\title{
Evaluación de los Programas de Formación en Cirugía G eneral. Encuesta a los alumnos de postítulo
}

\author{
Ricardo Espinoza $G^{1}$, Stefan Danilla $E^{1}$, Fabio Valdés $G^{1}$, \\ Ignacio San Francisco $R^{2}$, 0 svaldo Llanos $L^{2}$. \\ Resident evaluation \\ of general surgery training programs
}

Background: The profile of the general surgeon has changed, aiming to incorporate new skills and to develop new specialties. Aim: To assess the quality of postgraduate General Surgery training programs given by Chilean universities, the satisfaction of students and their preferences after finishing the training period. Ma terial and methods: A survey with multiple choice and Likert type questions was designed and applied to 77 surgery residents, corresponding to 59\% of all residents of general surgery specialization programs of Chilean universities. Results: Fifty five percent of residents financed with their own resources the specialization program. Thirty nine percent disagreed partially or totally with the objectives and rotations of programs. The opportunity to perform surgical interventions and the support by teachers was well evaluated. However, $23 \%$ revealed teacher maltreatment. Fifty six percent performed research activities, $73 \%$ expected to continue training in a derived specialty and $69 \%$ was satisfied with the training program. Conclusions: Residents considered that the quality and dedication of professors and financing of programs are issues that must be improved. The opportunity to perform surgical interventions, obtaining a salary for their work and teacher support are considered of utmost importance (Rev Méd Chile 2009; 137: 940-5).

(Key words: Education, medical, graduate; General surgery; Internship and Residency)

\begin{abstract}
Recibido el 11 de agosto, 2008. Aprobado el 30 de abril, 2009.
${ }_{1}^{1}$ Departamento de Cirugía, Facultad de Medicina, Universidad de los Andes. ${ }^{2}$ Departamento de Cirugía Digestiva, División de Cirugía, Facultad de Medicina, Pontificia Universidad Católica de Chile. Santiago de Chile.
\end{abstract}

L a especialización en cirugía ha experimentado, mundialmente, significativos cambios en las últimas dos décadas ${ }^{1-3}$. Por un lado se observa el mantenido desarmollo de las especialidades denivadas de la cirugía general; por otro, la cada vez mayor cantidad de conocimientos y habilidades necesarias para lograr una formación quinúrgica completa, que se deben entregar en un tiempo limitado $0^{4,5}$.

En el campo de la cirugía general, observamos que la formación primaria, a la que cada vez se suman nuevas competencias, se ha trasformado en

Correspondencia a: Dr. Ricardo Espinoza G. Teléfono: 4129307. Fax: 2141752. E mail: respinoza@uandes.cl un puente para acceder a especialidades derivadas $^{6}$. Lo anterior se puede traducir en un cambio del perfil del cirujano general y de su más inmediato ámbito de acción. En nuestro país, además, nos estamos incorporando a una cultura de acreditación de los programas docentes, con exigencias de estándares que permitan graduar especialistas competentes. No existen en Chile datos objetivos que permitan conocer cómo funcionan y cómo son valorados por sus propios alumnos los diferentes programas de formación en cirugía general. Recientemente, un grupo de trabajo de la Sociedad de Cirujanos de Chile y otro del Capítulo Chileno del Colegio Americano de Cirujanos ${ }^{7,8}$ han intentado definir cuáles son los requisitos y están- 
dares que debieran poseer los programas de especialización en cirugía general. Sin embargo, todavía no están disponibles todos los elementos que se deben considerar al momento de realizar una reforma en cuanto a la duración y los contenidos que definen los referidos programas.

Los objetivos del presente estudio fueron: conocer algunos aspectos sobre la calidad de los programas de formación en cirugía general que dan las universidades chilenas, determinar la satisfacción de los alumnos con sus respectivos programas y conocer sus preferencias una vez concluida su formación en cirugía general.

\section{MATERIAL Y MÉTODO}

Diseño. Para alcanzar los objetivos propuestos se comenzó con el diseño de una encuesta. Los ítems de la encuesta fueron generados por los autores del estudio, entre los que se cuentan docentes y alumnos de postítulo en cirugía general, y validados por una muestra independiente de otros alumnos de dicho postítulo, de modo de eliminar sesgos, tales como la ambigüedad de las preguntas y las preguntas con juicio de valor, entre otros. En este trabajo se denominarán indistintamente, alumnos de postítulo o residentes de cirugía a los médicos que cursan un Programa Universitario de Postítulo en Cirugía General.

Las preguntas de la encuesta se dividieron en tres dominios: generalidades, calidad del programa y preferencias después de terminar el Programa de Cirugía General. El primero y tercer dominios quedaron constituidos por preguntas de elección múltiple, 15 para el primero y cuatro para el tercero. El segundo dominio, referente a la calidad del programa, consistió en 51 ítems organizados en elección múltiple y escalas de Likert.

Universo. Se consideró como universo completo a la totalidad de los alumnos de los Programas de Formación en Cirugía General de todas las Facultades de Medicina de Chile, independiente del estado de acreditación del Programa y de la Facultad correspondiente. Para obtener respuesta se contactó individualmente a cada residente $\mathrm{y}$ al profesor encargado de cada uno de los Programas.

Estadística. Para el análisis, las variables de intervalo se describieron como mediana y rango; las variables ordinales (puntajes Likert) como porcentajes y las categónicas (elección múltiple) según ranking. La consistencia intema de la encuesta en las preguntas con escala Likert se midió con el alfa de Cronbach y la comelación entre ítems con mo $(\rho)$ de Spearman. Las comparaciones entre variables de intervalo entre dos grupos se realizaron con la prueba t de Student. Se empleó análisis de la vanianza para las comparaciones múltiples o sus equivalentes no paramétricos (MannWhitney y Kruskall-Wallis) si la distribución no era normal (Shapiro-Wilk) o si comespondían a variables ordinales. Las comparaciones de variables categónicas se realizaron con la prueba de $\mathrm{chi}^{2}$ o Fisher según comespondiera. Se consideró un nivel alfa de 5\% para todas las pruebas, excepto para las comparaciones múltiples donde se aplicó la comección de Bonfermoni.

Ética. Se garantizó la confidencialidad de los datos y no se incluyó información que permitiese identificar personalmente a cada respondedor de la encuesta. También se garantizó que las respuestas individuales o estratificadas por institución, no serían divulgadas.

\section{RESULTADOS}

\section{A. Generalidades}

De un universo de 131 residentes de Cirugía General en Chile durante el año 2007, obtuvimos 77 respuestas (59\%); 76,3\% de sexo masculino. Treinta residentes $(41,1 \%)$ cursaban el primer año de especialización, $30 \%$ el segundo y los restantes se encontraban en su último año. La edad promedio de los residentes al inicio del programa de especialización había sido 26 años. El 68\% se encontraba soltero al momento de la evaluación. La frecuencia de respuesta por universidad se aprecia en la Tabla 1.

Poco más de la mitad de ellos (55,3\%) financiaba su especialización con recursos propios y sólo un cuarto de la muestra $(23,7 \%)$ tenía financiamiento universitario. El 11,9\% estaba financiado por el Ministerio de Salud. De los encuestados, 47 (64,4\%) declaraba saber que su programa era de dedicación exclusiva y en esa condición, 36,2\% trabajaba en forma remunerada en forma simultánea a su residencia. Cuando no existía la restricción de exclusividad, 73,1\% trabajaba para financiarse.

En $12 \%$ de los casos los residentes no conocían el status de acreditación de su programa o sabían que no estaba acreditado. La mediana de las intervenciones quirúrgicas practicadas por los 
Tabla 1. Frecuencia de respuesta por universidad

\begin{tabular}{|lrr|}
\hline Universidad & $\mathbf{n}$ & $\%$ \\
\hline Universidad de Chile & 28 & 36,4 \\
Pontificia Universidad Católica de Chile & 21 & 27,3 \\
Universidad de los Andes & 9 & 11,7 \\
Universidad de Valparaíso & 7 & 9,1 \\
Universidad de Concepción & 5 & 6,5 \\
Universidad de Santiago & 4 & 5,2 \\
Universidad de la Frontera & 2 & 2,6 \\
Universidad del Desarrollo & 1 & 1,3 \\
Total & 77 & 100,0 \\
\hline
\end{tabular}

residentes al tercer año del programa era 530 como cirujano principal y 560 como ayudante, no existiendo diferencias significativas entre las distintas universidades. En 58\% de los casos, en los que el residente actuó como primer cirujano, el ayudante no fue un docente universitario, sino un cirujano de la planta asistencial del hospital.

\section{B. Calidad de la formación}

1. Programa. El $57,1 \%$ de los encuestados $(n=44)$ estuvo de acuerdo o totalmente de acuerdo en que los objetivos del Programa estaban claramente descritos y $88,4 \%$ en que las rotaciones correspondientes estaban bien definidas. Sin embargo, 30 de ellos (39\%) estuvo en desacuerdo o totalmente en desacuerdo en que los objetivos de cada rotación estuviesen claramente definidos. La formación se estimó insuficiente en trauma y urología. El 60\% eliminaría de los programas las rotaciones en neurocirugía y ginecología. Mirado en su globalidad, $60 \%$ de los residentes manifestó estar de acuerdo o completamente de acuerdo con la calidad del programa y $68,7 \%$ manifestó estar satisfecho con él, revelando una adecuada correlación, $\mathrm{p}<0,001$.

2. Docentes. En $40 \%$ hubo acuendo en que el número de docentes universitarios era el adecuado, otro $42,7 \%$ opinó que éste no era el que se requería. En $60 \%$ se evaluó como muy favorable la disponibilidad de los docentes para resolver dudas o dificultades. En $64,5 \%$ y $68,9 \%$ de los casos se evaluó como bueno o muy bueno el nivel de conocimientos y de destreza quirúrgica de los docentes, respectivamente. En 35\% se consideró buena la capacidad de investigación de los docentes. La relación de los residentes con los docentes fue calificada como buena o muy buena en 61\%, existiendo 14 testimonios (23\%) de mal trato y atropello frente a terceros. En $62,4 \%$ la relación entre los docentes fue observada como indiferente o mala. Entre los mismos residentes, en $74 \%$ las relaciones personales se calificaron como buenas o muy buenas.

3. Actividades docentes y evaluación. En la mitad de los casos los seminarios de análisis de temas quirúrgicos fueron bien evaluados $\mathrm{y}$ en igual proporción las visitas clínicas. El 41,6\% no estuvo de acuerdo con la forma como se evaluaron los conocimientos teóricos, y en 32,5\% los prácticos. El $59,2 \%$ de los residentes $(n=42)$ estuvo en desacuerdo o totalmente en desacuerdo con la cantidad de tiempo disponible para el estudio y $58 \%$ evaluó como no adecuada la relación entre la actividad docente y la asistencial. El 38\% de los encuestados refinió que entre la mitad y $75 \%$ de su tiempo activo desamolló actividades en pabellón quirúrgico.

4. Otros. De los residentes, 34 (44\%) declararon haber tenido oportunidad de participar en investigación básica y 6,7\% en un protocolo de tipo prospectivo. El 56,4\% (n =44) participó en investigación clínica. El 60\% y 80\% de los residentes reconoce falta de enseñanza en metodología de la investigación y en técnicas de docencia, respectivamente. Más de la mitad de los residentes no tuvo acceso a entrenamiento en escenarios no clínicos o a laboratorios de cirugía experimental. Un tercio de los residentes expresó estar de acuerdo con el sistema de financiamiento de su programa. En cuanto a la duración del programa, 31 (41,3\%) opinan que la duración debiera ser mayor. 
En la Tabla 2 se aprecia el orden de prioridades de los aspectos que los residentes mejorarían en su programa (moda 1 al 6; 6 mínima prioridad). Los elementos del programa que los encuestados consideran más importantes figuran en la Tabla 3, (moda 1 al 5; 5 mínima prioridad). La Tabla 4 muestra cuáles son los aspectos que los residentes agregarían a un programa de formación en cirugía general (moda 1 al 13; 13 mínima prioridad).

\section{Preferencias post-formación}

Concluida su formación como cirujano general, un poco más de un cuarto de los residentes $(27,3 \%)$ desea seguir ejerciendo la cirugía bajo esa forma. En ese caso, 62\% en regiones. Los restantes aspiran a una especialidad derivada de la cirugía, y en ese caso, 70\% desea trabajar en Santiago.

En la Tabla 5 se presentan, por orden de preferencia, las especialidades derivadas a las que postularían los residentes de cirugía general.

\section{DisCUSIÓN}

La estabilidad interna de la escala fue $91,6 \%$, por lo que se considera que el instrumento generado midió lo deseado en forma adecuada.
El perfil del residente de cirugía general es el de un profesional hombre, soltero, que ha iniciado su especialización a los 26 años de edad.

En este análisis nacional llama la atención que $68,7 \%$ de los residentes de un programa de cinugía general se muestra satisfecho de su formación, a pesar de que $40 \%$ de las respuestas señala aspectos que deben mejorarse. En este sentido son dos los temas que se resaltan: la dedicación de los docentes y el financiamiento. Si bien el número global de intervenciones quinúrgicas efectuadas durante el peníodo de residencia está de acuerdo con el estándar propuesto por el Comité de Estudio de Postgrado de la Sociedad de Cinujanos de Chile $^{7}$, no es menos cierto que en $58 \%$ de las veces en que un residente actúa como cirujano principal, es asistido por un cirujano quien no es docente universitario. Ello está dado por las características de los campos clínicos y escenarios de urgencia; hecho que adquiere relevancia si se analizan programas universitarios de formación de especialistas, que por la condición de ser universitarios, debieran contar con docentes en número adecuado. Esto mismo puede explicar la relativa baja frecuencia, que no sobrepasa $60 \%$ de los casos, en que los residentes efectúan investigación científica. Este punto es especialmente sensible a la hora de valorar críticamente un programa que por su esencia univer-

Tabla 2. Prioridades de mejoría en un Programa de Cirugía General

\begin{tabular}{|lc|}
\hline Item & Orden de prioridad \\
\hline Calidad y dedicación de los docentes & 1 \\
Financiamiento & 1 \\
Calidad y dedicación del jefe de programa & 3 \\
Disponibilidad de los docentes & 3 \\
Calidad del centro formador & 4 \\
Selección de los residentes & 5 \\
Duración del programa & 6 \\
\hline
\end{tabular}

Prioridad señalada con mayor frecuencia (moda) por los encuestados, según ítem.

Tabla 3. Factores de importancia en un Programa de Cirugía G eneral

\begin{tabular}{|lc|}
\hline Factor & Orden de factores \\
\hline Oportunidad de operar & 1 \\
Remuneración económica & 1 \\
Apoyo docente & 1 \\
Facilidades para estudiar & 3 \\
Respaldo legal & 5 \\
Posibilidades para investigar & 5 \\
\hline
\end{tabular}

Prioridad señalada con mayor frecuencia (moda) por los encuestados, según ítem. 
Tabla 4. Temas a incorporar en un Programa de Cirugía G eneral

\begin{tabular}{|lc|}
\hline Tema & O rden de temas \\
\hline Cirugía experimental & 1 \\
Laboratorios de simulación laparoscópica & 1 \\
Medicina basada en la evidencia & 1 \\
Metodología de la investigación & 4 \\
Idiomas & 5 \\
Epidemiología clínica & 6 \\
Bioestadística & 6 \\
Investigación en ciencias básicas & 7 \\
Administración y gestión & 9 \\
Derecho sanitario & 12 \\
Técnicas de docencia & 12 \\
Evaluación económica en salud & 13 \\
Deporte & 13 \\
\hline
\end{tabular}

Prioridad señalada con mayor frecuencia (moda) por los encuestados, según ítem.

Tabla 5. Especialidades derivadas preferentes concluida Cirugía General

\begin{tabular}{|lrr|}
\hline Especialidad derivada & $\mathbf{n}$ & $\%$ \\
\hline Cirugía plástica, reparadora y estética & 19 & 32,2 \\
Digestivo alto, hígado y biliopancreático & 8 & 14,8 \\
Cirugía vascular & 5 & 9,3 \\
Cirugía de tórax & 5 & 9,3 \\
Trasplante & 1 & 1,9 \\
Coloproctología & 5 & 9,3 \\
Urología & 3 & 5,6 \\
Cirugía de cabeza y cuello & 3 & 5,6 \\
Cardiocirugía & 3 & 5,6 \\
Cirugía oncológica & 2 & 3,7 \\
\hline
\end{tabular}

sitaria debiera, también, destacarse por esta actividad. En forma coherente, entre los temas que los residentes estiman que deben incorporarse a los programas, surge con fuerza la lectura crítica de la literatura y la metodología de la investigación, como hemamientas que apuntan a una mejońa cualitativa. Efectivamente, las bases científicas de la cirugía han alcanzado tal profundidad y las estrategias de manejo de la patología quinúrgica hoy son tan complejas, que se requiere formación adicional en esos aspectos para lograr una sólida formación del especialista, ${ }^{9,10}$.

El otro tema relevante para los residentes es el económico. Sólo un poco más de un tercio de ellos cuenta con financiamiento, y la gran mayoría realiza actividades paralelas al programa. Es conocido el efecto negativo que este hecho tiene sobre la formación, fundamentalmente por la distracción de tiempo y dedicación, en un programa que ya es reducido en su duración. Recordemos que en Estados Unidos de Norteamérica y países europeos los programas tienen una duración de cinco años, y son remunerados. Eso asegura una dedicación completa al programa de especialización ${ }^{11}$.

La referencia a la necesidad de entrenamiento en escenarios no clínicos apunta también a lograr que el residente pueda resolver adecuadamente diversas competencias que se aprenden a través de la práctica repetida de ellas, o aquellas que, por su baja frecuencia de presentación, no permiten un enfrentamiento cotidiano ${ }^{12,13}$. El cinujano se forma a través de un esfuerzo sostenido en un programa que le entregue una sólida formación científica y las posibilidades de desarmollar las destrezas propias de la especialidad. Como se mencionó, ante una restricción horaria a la dedicación, que en nuestro medio no ha alcanzado aún una reglamentación objetiva y la 
negativa evaluación social, o la cada vez más difícil aceptación que hoy tiene el entrenamiento en pacientes, sin la exposición previa a modelos no animados, es que el entrenamiento quirúrgico puede recumir en la actualidad, al menos en parte, a recursos docentes fuera de la sala de operaciones ${ }^{12}$. La simulación es una estrategia pedagógica efectiva cuando se quiere enseñar competencias, evitar y comegir emores ${ }^{14,15} \mathrm{y}$ se perfila, por tanto, como una meta por implementar.

Por otro lado, si bien uno de los objetivos de una residencia quinúrgica es capacitarse para efectuar la intervención apropiada, en el momento adecuado y realizarla bien, la educación quinúrgica es más que eso. Aparte de la incorporación de los conocimientos necesarios, se trata de transmitir una actitud de vida y de la valoración del paciente con todo el profesionalismo necesario ${ }^{4}$. Por ello es que también en cirugía, como en todas las disciplinas médicas, el curriculum oculto y la poderosa transmisión de valores de manera no escrita, son asuntos de vital importancia. La información entregada por esta encuesta referente al trato entre cinujanos y los residentes es una seria advertencia a los aspectos cualitativos de la docencia. En nuestro medio existen datos sobre conductas abusivas en educación médica a nivel de pregrado ${ }^{16}$ y nuestro reporte de $23 \%$ de trato humillante de un

\section{REFERENCIAS}

1. Peliegrini CA, Warshaw AL, Debas HT. Residency training in surgery in the $21^{\text {st }}$ century: A new paradigm. Surgery 2004; 136: 953-65.

2. Peimegrin CA. Surgical Education in the United States. Navigation the White Waters. Ann Surg 2006; 244: 335-42.

3. SACHDEVA AK. The Changing Paradigm of Residency Education in Surgery: A Perspective from the American College of Surgeons. Am Surg 2007; 73: 120-9.

4. Stern DT, PaPADAKIS M. The Developing PhysicianBecaming a Profesional. NEngl J Med 2006; 355: 1794-9.

5. CORE COMMTTTE INSTITUTE FOR INTERNATIONAL MEDICAL EDUCATION. Global minimum essential requirements in medical education. Medical Teacher 2002; 24: 130-5.

6. Whaien TV. Duty Hours Restrictions: How Will this Affect the Surgeon of the Future? Am Surg 2007; 73: 140-2.

7. Hepp J, CSEndes A, Ibáñez F, Llanos O, San Martín S. Programa de la especialidad Cinugía General. Definiciones y propuestas de la Sociedad de Cinujanos de Chile. Rev Chil Cir 2008; 60: 79-85.

8. Llanos O, San Martín S, Calderón W, Boza C. Requisitos mínimos y estándares básicos de un programa de formación de un cirujano general. Rev Chil Cir 2003; 55: $655-8$. docente a un alumno de postítulo es un hecho grave. Dado el poderoso efecto modelo de un profesor, las actitudes abusivas de éste tienen reconocidos efectos sobre los estándares de conducta y de práctica de quienes están en formación.

Para concluir, el hecho que dos tercios de nuestros residentes deseen desamollar una especialidad denivada luego de la cinugía general, se acerca a las observaciones de Norteamérica ${ }^{1}$ en que cerca de $75 \%$ de los egresados de estos programas desea completar su formación en un área específica. Por último, pese a la complejidad que ha alcanzado la cinugía, la cirugía general sigue vigente, con los cambios propios del progreso, no sólo como una etapa en la formación de los especialistas quinúrgicos, sino también como una especialidad en sí misma, necesaria para cubrir las necesidades de hospitales generales y mantener los cuidados quinúrgicos básicos que, a su vez, sostienen a las especialidades derivadas.

\section{Agradecimientos}

Los autores agradecen la colaboración prestada por todos aquellos residentes de los programas de cirugía general que contestaron la encuesta e hicieron posible la realización del presente estudio. Mención especial al Dr. Alexandre Sauré M. por su ayuda en la digitalización de los datos.

9. BASS BL Fundamental Changes in General Surgery Residency Training. Am Surg 2007; 73: 109-13.

10. Espinoza R. Cirugía y Docencia. Rev Chil Cir 2005; 57: 365.

11. Parrila P, Landa Ji, Moreno-Gonzáiez E, Aiarco A, Martínez E, Rodríguzz JA et al Proyecto de programa de la especialidad de cirugía general y del aparato digestivo. Cir Esp 2006; 80: 133-44.

12. BurNs RP, BURKHOLDER HC. Simulated Surgical Skills Training: Modem-Day Surgical Homework. Am Surg 2007; 73: 166-9.

13. Seymour NE, Galiaher AG, Roman SA, O'Brien MK, BANSAL VK, ANDERSEN DK et al. Virtual Reality Training Improves Operating Room Performance. Ann Surg 2002; 236: 458-64.

14. REzNICK RK, MaCrae H. Teaching Surgical Skills- Changes in the Wind. NEngl J Med 2006; 355: 2664-9.

15. Moorthy K, Munz Y, ForRest D, Pandey V, Undre S, VINCENT C et AL Surgical Crisis Management Skills Training and Assessment. A Stimulation-Based Approach to Enhancing Operating Room Performance. Ann Surg 2006; 244: 139-47.

16. Maida AM, Herskovic V, Pereira S, Salinas-Fernández L, Esquvel C. Percepción de conductas abusivas en estudiantes de medicina. Rev Méd Chile 2006; 134: 1516-23. 